\title{
Case Report: Treating Severe Atrophic Maxillary Arch with Horizontal Ridge Augmentation
}

\section{Kavish A Gurjar \\ State of the Art Dental Group, Rockville,} MD, 20850, USA
Correspondence: Kavish A Gurjar State of the Art Dental Group, Rockville, MD, 20855, USA

Tel + | 8187465914

Email Drkavishagurjar@gmail.com
Abstract: A deficient maxillary edentulous ridge volume can be augmented by onlay grafting technique for implant placement to support fixed hybrid screw retained prosthesis. Various techniques have been used to correct defective bone. These augmentation techniques include inlay and onlay grafts, bone splitting osteotomy, and distraction osteogenesis, among others. Successful reconstruction depends on multiple biological factors as well as patient compliance. Here, I report a case of ridge augmentation using onlay cortico-cancellous particulate bone grafting, resorbable collagen membrane, and bone tac pins, followed by surgical implant placement with multiunit abutments to support the fixed prosthesis.

Keywords: atrophy, medullary bleeding, dental implant, ridge augmentation, bone grafting, guided bone regeneration, GBR, collagen membrane

\section{Introduction}

A patient requiring dental treatment can present with several underlying diseases and/or disorders. Therefore, it is the clinician's responsibility to gather information about the presenting patient's oral and systemic conditions to ensure appropriate treatment with a successful outcome. Many patients with a history of multiple extractions and who wear partial or complete dentures may have severely atrophic alveolar ridges. These patients may seek dental treatment to obtain improved mastication and a better quality of life.

Bone augmentation of an atrophic maxilla can increase the available bone for endosseous implant-supported fixed screw-retained prosthesis. Patients with severe atrophy of the maxilla can be treated with onlay allograft to increase the ridge volume, which can further be used to place dental implants and fixed prosthesis to restore stability and bite function. Allografts have several advantages; one of them is that it causes no harm to the donor area, which is usually reported with autograft or block graft using hip bone or chin bone. Further, patients do not require hospitalization like for augmentation with autograft from hip bone. Onlay grafting can be done when certain sites cannot achieve augmentation by ridge splitting for 1-mm-thick bone predictively. One disadvantage of onlay grafting is that patient will have to be extra cautious with a strict soft diet and use removable prosthesis that may apply pressure on the augmentation site causing resorption, ultimately affecting transient function until the fixed restoration is provided. The patient has to be on semi-solid and soft diet to prevent any pressure on the ridge augmentation site. Alternatively, we can use block-grafting or ridge splitting technique to perform ridge augmentation. Ridge splitting technique is not possible when a certain area or ridge is $1-\mathrm{mm}$ thick. 
This report describes the treatment of horizontal ridge augmentation of maxillary arch success, which can help restore function by maxillary implant-supported fixed screw-retained prosthesis.

\section{Case Report}

The patient was a 55-year-old man whose chief concern was "I want to chew food, and I do not want to wear dentures." The patient was prepared in the usual manner for dental implant treatment, which included diagnostic casts, a clinical oral visual exam, cone-beam computed tomography $(\mathrm{CBCT})$, and a review of his medical and

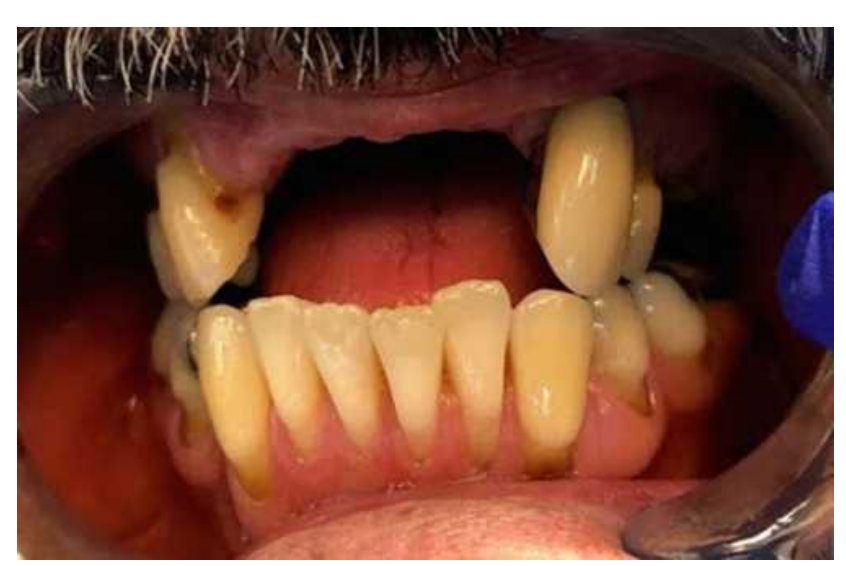

Figure I Preoperative anterior maxillary atrophy. dental history. The patient was in the army for 10 years and had poor dental care which caused significant dental caries; the affected teeth needed to be extracted owing to limited dental treatment options while on duty. The patient was a non-smoker and did not have diabetes or any other known medical conditions or allergies.

The maxillary teeth were found to be carious with poor periodontal prognoses, and the maxillary area was partially edentulous with severe osseous atrophy (Figure 1). The anterior maxillary ridge bone was 1-2-mm thick (facial/lingual) on CBCT (Figure 2). Treatment options were discussed with the patient, and written informed consent was obtained.

The treatment plan included extraction of the remaining teeth, maxillary lateral (horizontal) osseous particulate graft augmentation, and subsequent implant placement to support a screw-retained fixed hybrid prosthesis.

The patient was prescribed $400 \mathrm{mg}$ etodolac (Lodine) as anti-inflammatory medication and $875 \mathrm{mg}$ amoxicillin/ clavulanic acid (Augmentin) as the antibiotic agent, to be started two days prior to surgery, and to continue the drug seven days postoperatively. Two days prior to the surgery date, the patient came in for an emergency dental visit for lingering pain and infection in the upper right canine. To maintain the scheduled surgery date, the patient was prescribed antibiotics and pain killers.

The patient presented on the day of surgery, and a $20 \%$ benzocaine topical gel was applied (NIVO industries, Inc.,

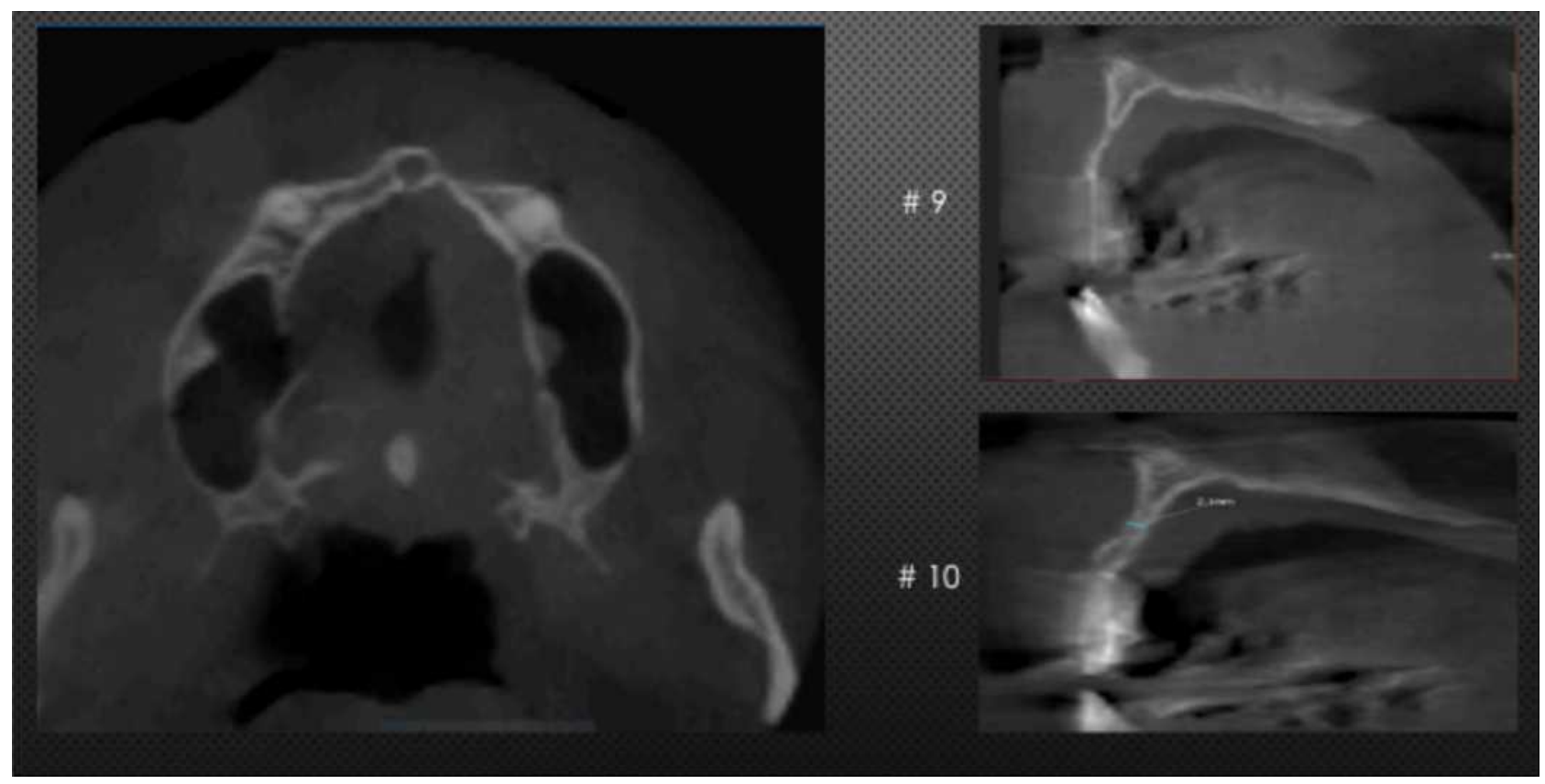

Figure 2 Preoperative CT. 
Santa Monica, CA). Local infiltration anesthetization was performed using three carpules of $2 \%$ lidocaine $\mathrm{HCl}$ $(1.7 \mathrm{~mL})$ and epinephrine $(1: 100,000)$ injection and three carpules of a $4 \%$ articaine $\mathrm{HCl}$ (Septocaine) and epinephrine 1:100,000 (1.7 mL) injection (Septodont France; Novocol Pharmaceuticals of Canada, Inc., Cambridge, Ontario). No sedation was administered. An immediate complete denture had been prefabricated at previous appointments. The remaining teeth were simply extracted, and the sockets were curetted and scrupulously debrided using a No. 8 round burr and hand curettes with copious irrigation with normal saline. A second CBCT was performed intraoperatively. A full thickness facial periosteal flap was raised from the first molar site to the contralateral first molar site. Buccal releasing incisions were placed at the first molar sites. Cortical perforations were made with a No. 4 round burr for access to subjacent cancellous bone and to achieve medullary bleeding (Figure 3). Periosteal scoring was performed in the sulcular area by a releasing incision with a No. 15 blade to achieve primary closure after bone graft placement. Subsequently, this area was covered with a collagen barrier membrane (BioMend Extend Membrane; Zimmer Biomet Dental, 4555 Riverside Drive Palm Beach Gardens, FL, USA). The barrier was press-fit bone tacked into position with a nonresorbable titanium bone tac

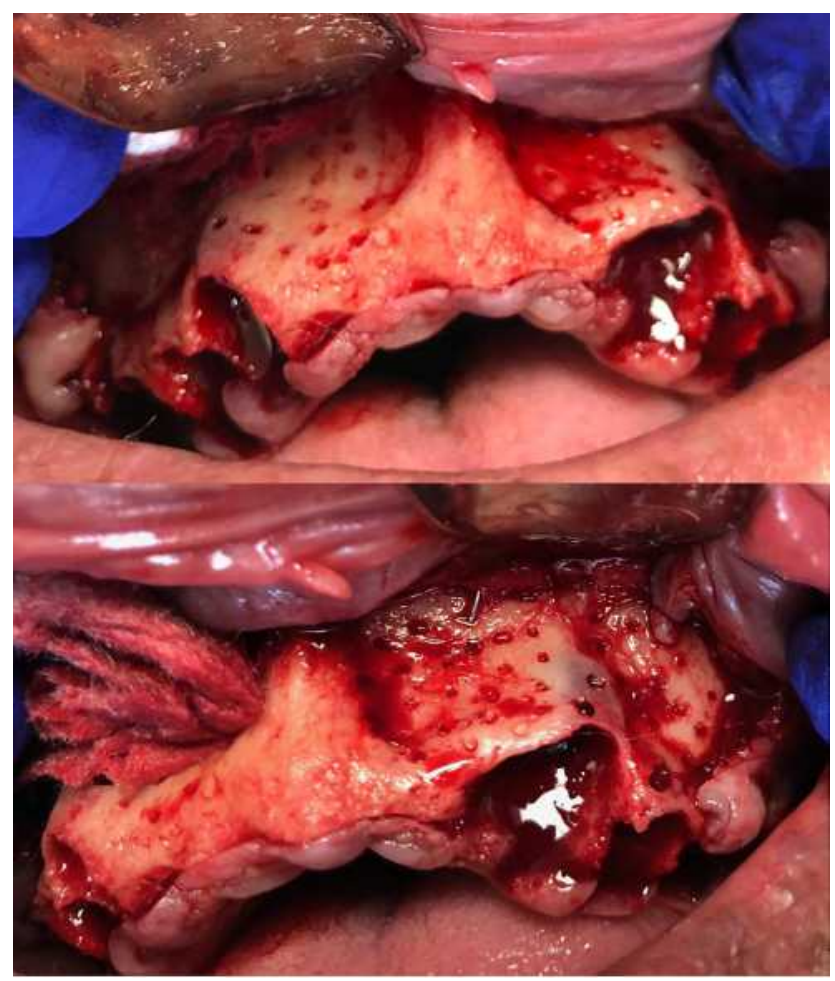

Figure 3 Decortication for medullary bleeding. measuring $3 \mathrm{~mm}$ in length pins using mallets on the facial and apical aspects (Figure 4). A total of four bone tacs pins were used on the right side and two on the left side. A $15 \mathrm{cc}$ BioHorizons MinerOss (Birmingham, Al.) corticocancellous allograft bone graft material was placed at the maxillary facial and crestal osseous aspect and extraction socket area (Figure 4). Following this, the palatal aspect of the membrane was secured, and a primary flap closure was performed using a combination of simple interrupted and continuous 3-0 black silk sutures to obtain primary closure (Figure 5). A third CBCT was obtained postoperatively

An immediate temporary removable acrylic complete denture was delivered and adjusted for fit and immediately soft-relined. The patient was advised to be on a complete soft diet for 4 months postsurgery. The complete removable temporary denture was used to maintain the vertical dimension of occlusion.

Written and verbal postoperative instructions and prescriptions for amoxicillin and clavulanate $(875 \mathrm{mg}$, bid) and chlorhexidine oral rinse (bid) were given to the

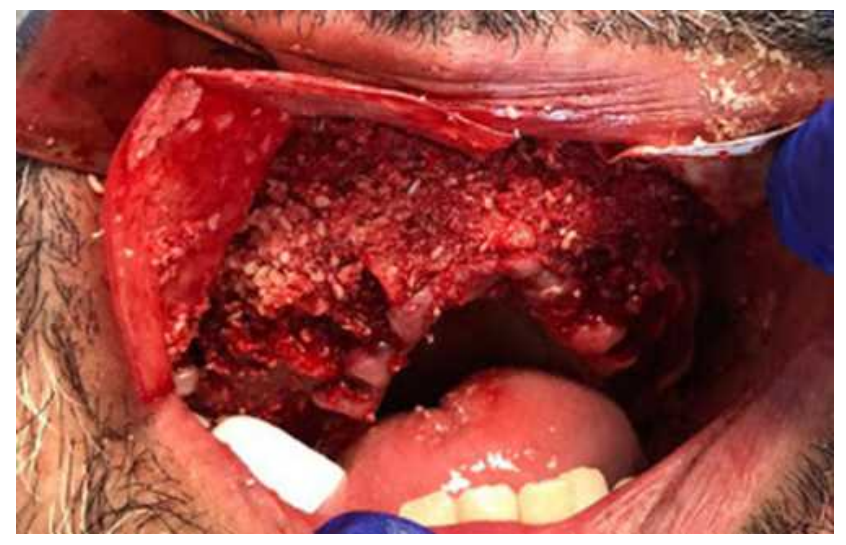

Figure 4 Particulate grafting with collagen membrane.

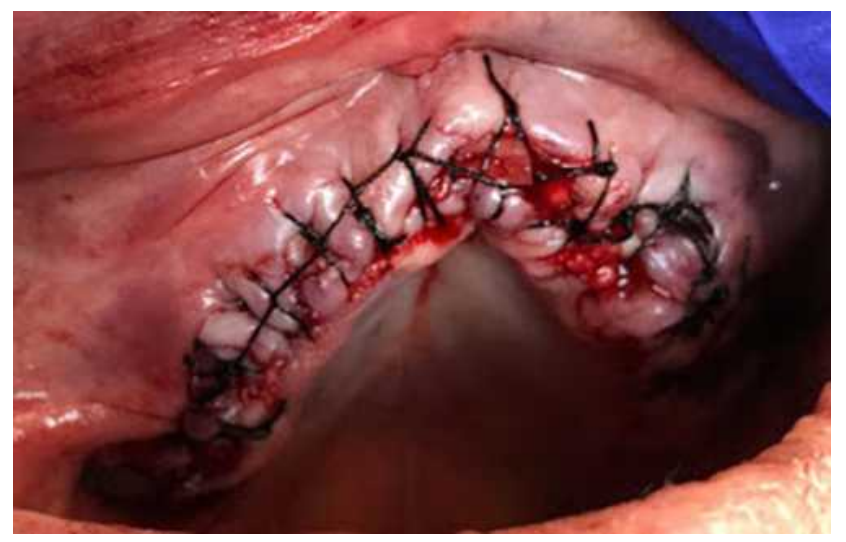

Figure 5 Primary closure with suture. 
patient. Etodolac (Lodine, $400 \mathrm{mg}$ ) was continued every 6 $\mathrm{h}$ as needed for pain control and anti-inflammation.

The patient returned for silk-suture removal after two weeks and underwent routine follow-up visits. After four months, a fourth CBCT was obtained to evaluate osseous healing. The maxillary ridge was found to be augmented to 6-8 $\mathrm{mm}$ in width as compared to the $1-2 \mathrm{~mm}$ of original thickness (Figure 6). The number of Hounsfield units was 600 , indicating relatively dense bone. Width was increased remarkably, which was necessary and adequate for implant placement (Figure 7).

After 4 months, a polyvinyl siloxane impression was taken, and a fifth CBCT was obtained using radiographic CT23 markers placed on the facial and palatal aspects of

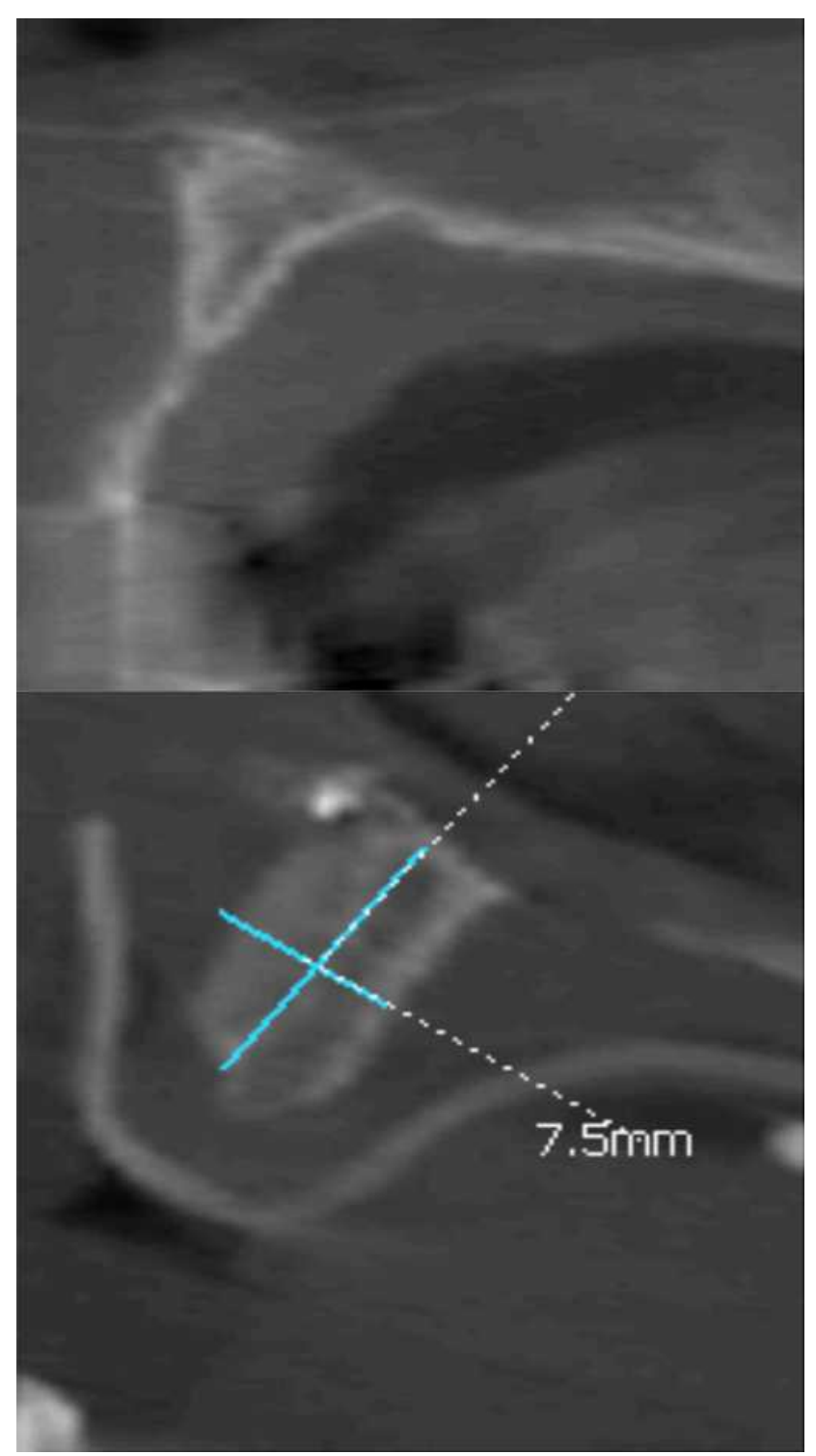

Figure 6 Postoperative. the denture for dual scans. CT markers are stickers that reflect radiopacity on a CT scan and are used to locate the positioning on a CT scan; this method was used to superimpose the scan without the denture for more accuracy. Utilizing this scan, digitally fully guided implant planning was done for BioHorizons implants placement.

Implant placement surgery was performed 4 months after bone graft placement (Figure 8). On the day of surgery, benzocaine $20 \%$ topical gel was applied (NIVO Industries), Monica, CA) followed by local infiltration anesthetization with three carpules of $2 \%$ lidocaine $\mathrm{HCl}(1.7 \mathrm{~mL})$ and epinephrine $(1: 100,000)$ injection and 3 three carpules of $4 \%$ Septocaine (articaine $\mathrm{HCl}$ (Septocaine) $4 \%$ and epinephrine $(1: 100,000)(1.7 \mathrm{~mL})$ injection (Septodont France, manufactured by Novocol Pharmaceuticals of Canada, Inc., Cambridge, Ontario N1T6X3). The patient was not anxious, and thus sedation was not required. A full arch tissuesupported fully guided surgery template was fabricated by a local dental laboratory and was secured against the maxilla with three screws (BioHorizons, Birmingham, Al). Osteotomies were performed, and the implants were installed, using 5 BioHorizons implant: Laser-Lok (Birmingham, AL, 35244 USA) (Table 1). Primary closure was performed using silk sutures. After implant placement, using 5 temporary titanium sleeves, 5 multiunit abutment analogs, and 5 multiunit abutments (BioHhorizons, Birmingham, Al) (Table 2), a screw-retained fixed acrylic prosthesis was fabricated (prefabricated during previous appointments), and an attempt was made to maintain the vertical and centric relation; chair side conversion of the denture was performed with soft relining for an immediate load (Figure 9). The occlusal plane was maintained with point contact, and no lateral interferences were present. A sixth CBCT was performed post-operatively. Owing to different angulation, multi-unit abutments were used for parallelism and stability of the occlusal plane. Written and verbal postoperative instructions were given to the patient, with prescriptions for amoxicillin and clavulanate $(875 \mathrm{mg}$, bid) and chlorhexidine oral rinse (bid). Etodolac (Lodine) $400 \mathrm{mg}$ was prescribed every six hours as needed for pain control and anti-inflammation, which had begun two days prior to the procedure, was continued for 7 more postoperative days. The patient returned after two weeks for silk-suture removal.

The treatment course was uneventful for more than 6 months. The patient presented for routine maintenance and adjustments and subsequently resumed his normal diet after this procedure with no further restrictions. 


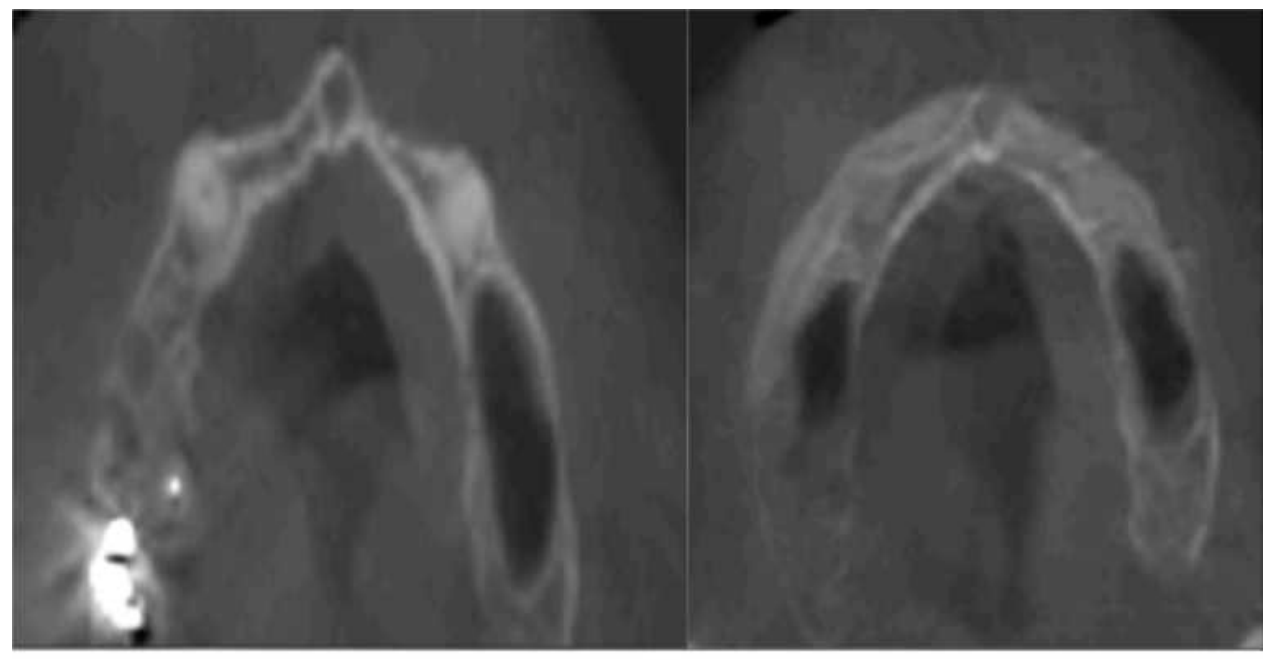

A

B

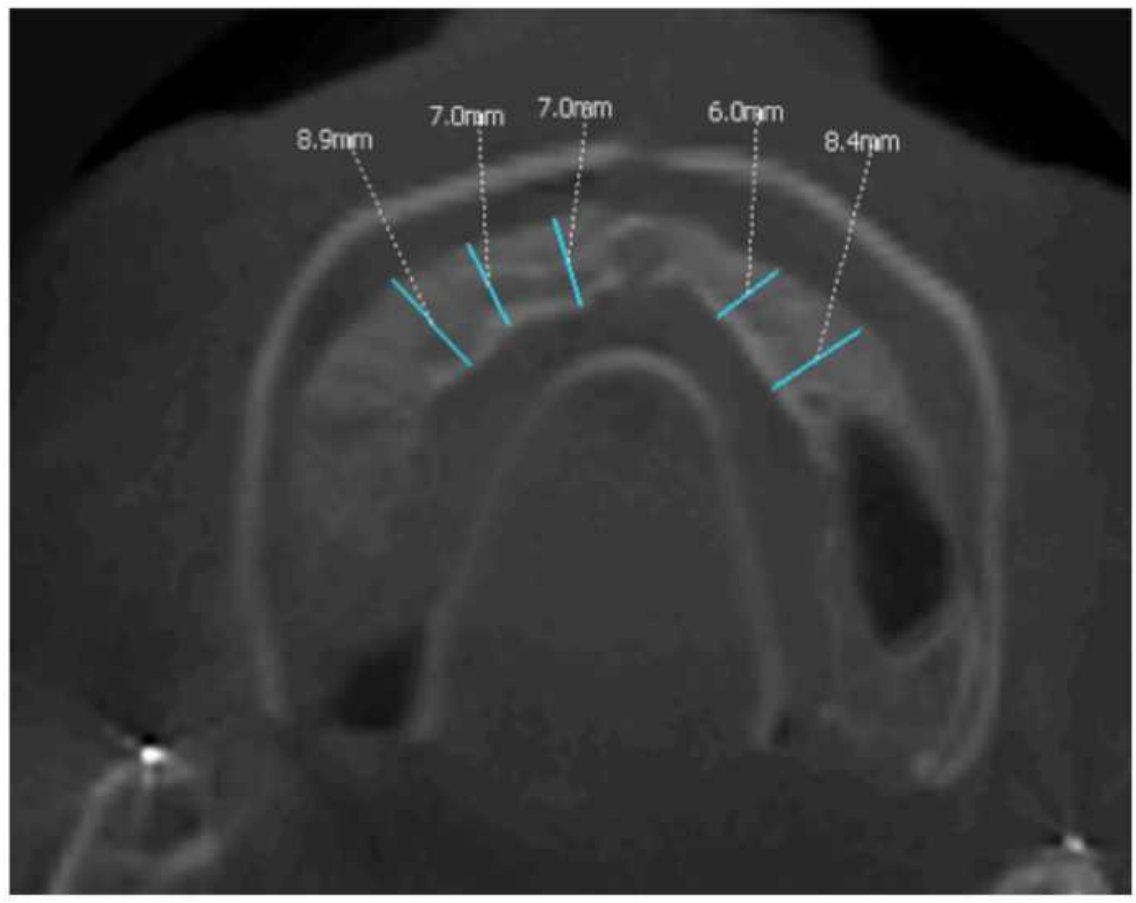

C

Figure 7 CT after 4 months: (A) preoperative width, (B) postoperative change in width, (C) measurements of postoperative augmentation.

\section{Discussion}

This patient had very severe atrophy with a $1-2-\mathrm{mm}$ thick maxillary ridge bone, which narrowed the available treatment methods to augment the ridge. Bone loss was due to multiple teeth loss due to the patient being on army duty with poor dental hygiene follow-up. This could be avoided by maintaining regular hygiene appointments. Ridge augmentation is a very technique-sensitive procedure. The option of a ridge split was not a good option in this case given the extremely thin bone. Therefore, the decision was made to extract the remaining teeth and perform guided bone regeneration (GBR) using a particulate corticocancellous bone graft. The Biomend Extend firm resorbable collagen membrane was secured with tenting pins. ${ }^{1,7}$ The patient also wanted to wear a denture, which was another challenge to the success of the procedure, because while the 

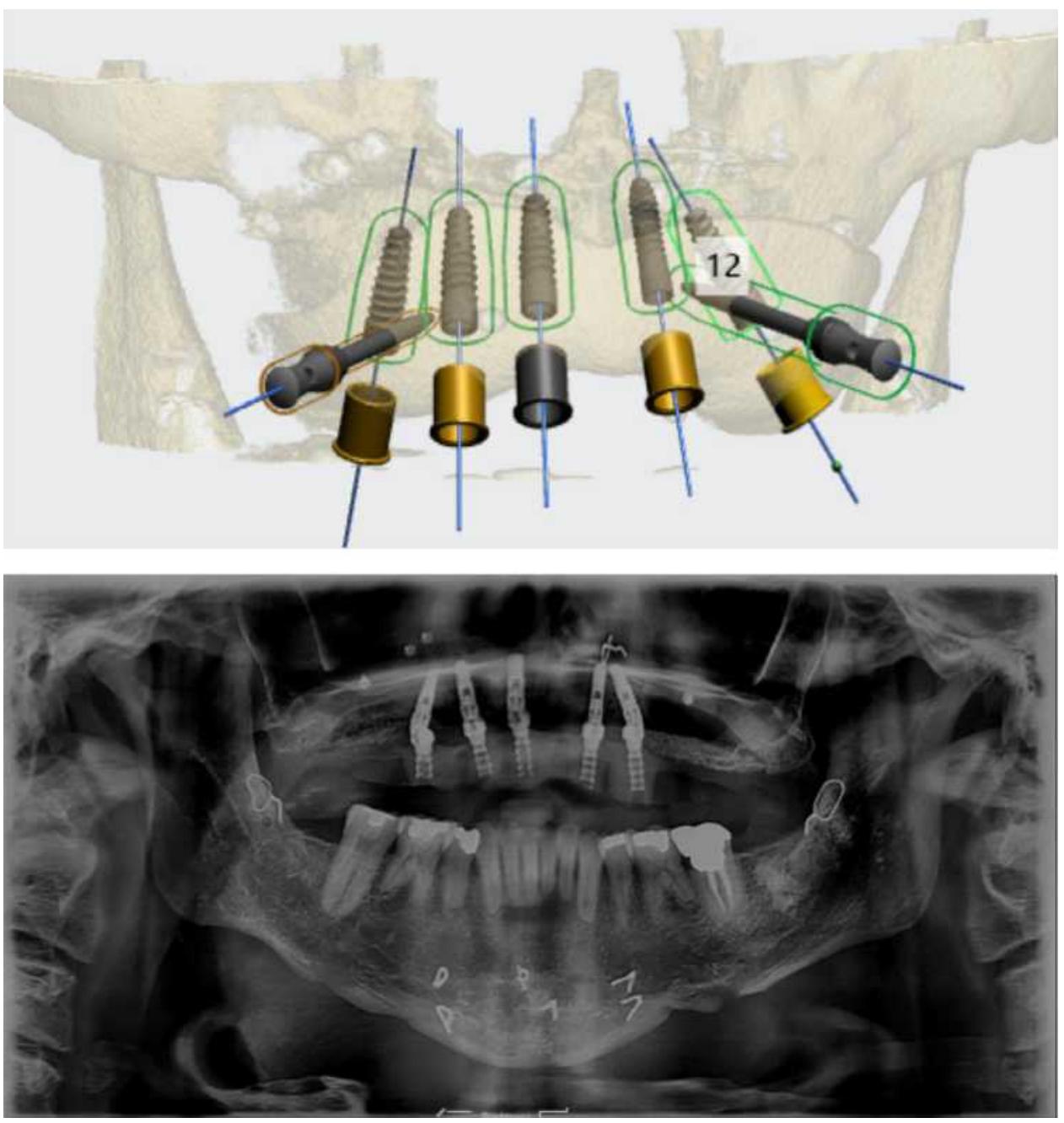

Figure 8 Digital planning and post-implant placement.

denture is worn, the flange of the denture can cause pressure resorption of the graft or graft failure. The success of this procedure is mainly dependent on two factors that can be controlled: one is successfully performing the surgery due to technique sensitivity, and the second is patient maintaining compliance with all the postoperative instructions.

Table I 5 BioHorizons Implant: Laser-Lok (Birmingham, AL 35244 USA)

\begin{tabular}{|l|l|l|c|}
\hline Implant Site & Length & Diameter & Platform \\
\hline$\# 3$ & $12 \mathrm{~mm}$ & $3.8 \mathrm{~mm}$ & $3.5 \mathrm{~mm}$ \\
\hline$\# 5$ & $12 \mathrm{~mm}$ & $3.8 \mathrm{~mm}$ & $3.5 \mathrm{~mm}$ \\
\hline$\# 7$ & $12 \mathrm{~mm}$ & $3.8 \mathrm{~mm}$ & $3.5 \mathrm{~mm}$ \\
\hline$\# 10$ & $12 \mathrm{~mm}$ & $3.0 \mathrm{~mm}$ & $3.0 \mathrm{~mm}$ \\
\hline$\# 13$ & $12 \mathrm{~mm}$ & $3.8 \mathrm{~mm}$ & $3.5 \mathrm{~mm}$ \\
\hline
\end{tabular}

After surgery, home care is an important factor, because the patient needs to receive follow-up home care for up to 4 months, including the following restrictions: eating a soft diet, not whistling, not using a straw, removing the denture(s) every night, not eating chewy foods, and not wearing the denture(s) while doing any physical exercise.

Table 2 Multiunit Abutment (BioHhorizons, Birmingham, Al) Size

\begin{tabular}{|l|c|l|l|}
\hline Implant Site & Gingival Height & Angle & Platform \\
\hline$\# 3$ & $2.25 \mathrm{~mm}$ & $17^{\circ}$ & $3.5 \mathrm{~mm}$ \\
\hline$\# 5$ & $2.25 \mathrm{~mm}$ & $17^{\circ}$ & $3.5 \mathrm{~mm}$ \\
\hline$\# 7$ & $2.25 \mathrm{~mm}$ & $17^{\circ}$ & $3.5 \mathrm{~mm}$ \\
\hline$\# 10$ & $2.25 \mathrm{~mm}$ & $17^{\circ}$ & $3.0 \mathrm{~mm}$ \\
\hline$\# 13$ & $2.25 \mathrm{~mm}$ & $17^{\circ}$ & $3.5 \mathrm{~mm}$ \\
\hline
\end{tabular}



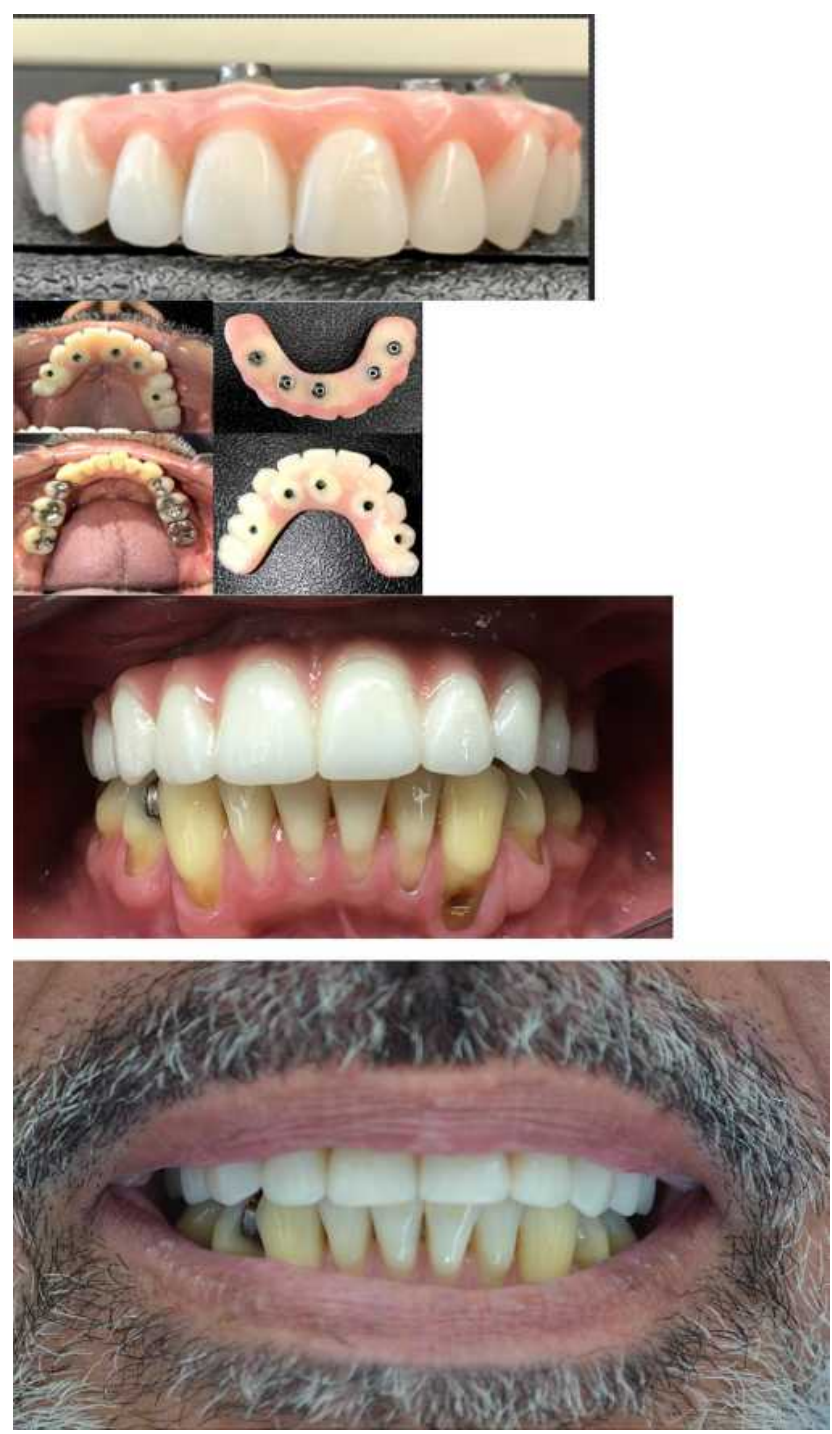

Figure 9 Hybrid prosthesis.

Alveolar ridge defects can occur because of many factors such as tooth loss, infection, trauma, and fractures. Such a defect compromises the implant stability and affects the ideal placement of the implants. Lateral and vertical ridge augmentation can be achieved by using a cortico-cancellous allograft, which is better for patients and does not cause traumatic surgery using autografts from the hip and ramus. Ridge augmentation is advised for restoring the structure, function, and stability of bite.

We used allografts with resorbable BioMend Extend membranes secured with 3-mm non-resorbable titanium bone tac pins that was never removed to prevent second surgery. In vitro studies comparing resorbable collagen membranes and nonresorbable membranes have demonstrated that bioresorbable membranes have a better osteoblastic potential and suggest better bone formation than nonresorbable membranes. ${ }^{7}$ Absorbable collagen membranes have cell-occlusive characteristics, serve as barriers to prevent epithelial cell migration, and allow the passage of essential nutrients. ${ }^{7}$ In addition, such membranes eliminate second-stage surgery for membrane removal, are designed to reduce wound trauma and surgical chair time, and are sufficiently rigid scaffolds for tissue regeneration in guided tissue regeneration (GTR) and GBR procedures. ${ }^{7}$ Furthermore, the membranes are tear resistant, suturable, pliable, and easy to handle even when hydrated. The BioMend Extend membrane is designed to be absorbed in approximately 18 weeks, providing enough time to stabilize the bone graft. The CopiOs extend membrane membrane (Zimmer Biomet Dental: 4555 Riverside Drive Palm Beach Gardens, Florida 33410) has a similar firmness suitable for managing this type of case. Cortical bone takes time to turn over and thus gives the cancellous part of the bone graft time to mature and stabilize eventually, causing less resorption and collapse of graft volume.

A Meisinger Pin-Control Membrane Fixation Kit was used to secure the membrane with titanium pins with a mallet. In step 1, a full thickness flap is reflected from molar to contralateral molar, maintaining the reflection of the flap and periosteal scoring by using a No. 15 blade to achieve primary flap closure. This is an important step to ensure that the bone and resorbable collagen membrane are not exposed and to thereby achieve successful GBR. In step 2, the membrane is secured with bone tacs, and decortication is performed on the labial aspect after a thorough curettage to achieve medullary bleeding. This is necessary to ensure that the blood supply is initiated for the graft from the recipient bed. In step 3, the corticocancellous bone graft is installed, and in step 4, there is primary closure of the flap using monofilament or silk sutures with a combination of an interrupted and horizontal mattress. Securing the membrane before placing the bone graft is one of the most important aspects for the procedure to be efficient and effective. ${ }^{2}$

Various bone grafts have been studied using only cortical grafts, only cancellous grafts, or a combination of cortical and cancellous grafts. ${ }^{3,4}$ Cortical grafts need to provide structure and volume for the loss of the alveolar ridge, and cancellous grafts are needed for more blood flow and a porous structure that can allow medullary bleeding, which can help maintain vitality and reduce graft rejection on the most outer part. ${ }^{1}$ This suggests that using a combination of cortico-cancellous grafts provides 
vitality as well as structure and stability. ${ }^{2-4}$ Autografts have recognized limitations such as donor site morbidity, potential resorption, size mismatch, and an inadequate volume of graft material. $^{8}$

This case report demonstrates the need for appropriate preoperative information gathering to accomplish a successful outcome and helps rule-out patients who are immunocompromised, smokers, or have uncontrolled diabetes. Compared to autografts, allografts provide a great means to restore bone with stable occlusion, require less maintenance (donor site morbidity), and result in great patient satisfaction. ${ }^{5,6}$

\section{Conclusion}

Preoperative information on the patient's presenting condition ensures a successful treatment outcome with longterm uneventful functionality. Osseous augmentation for bone volume and quality is essential. This patient was successfully treated with a maxillary implant-supported fixed screw retained zirconium prosthesis.

\section{Institutional Approval Statement}

No institutional approval was required to publish this case report.

\section{Patient Written Informed Consent Statement}

Written informed consent and verbal consent was provided by the patient to use case details and all accompanying images related to the case for publication.

\section{Disclosure}

The author reports no conflicts of interest in this work.

\section{References}

1. Dasmah A, Thor A, Ekestubbe A, Sennerby L, Rasmusson L. Particulate vs. block bone grafts: three-dimensional changes in graft volume after reconstruction of the atrophic maxilla, a 2-year radiographic follow-up. J Craniomaxillofac Surg. 2012;40(8):654-659. doi:10.1016/j.jcms.2011.10.032

2. Simion M, Fontana F, Rasperini G, Maiorana C. Vertical ridge augmentation by expanded-polytetrafluoroethylene membrane and a combination of intraoral autogenous bone graft and deproteinized anorganic bovine bone (Bio Oss). Clin Oral Implants Res. 2007;18 (5):620-629. doi:10.1111/j.1600-0501.2007.01389.x

3. Fennis JP, Stoelinga PJ, Jansen JA. Mandibular reconstruction: a histological and histomorphometric study on the use of autogenous scaffolds, particulate cortico-cancellous bone grafts and platelet rich plasma in goats. Int J Oral Maxillofac Surg. 2004;33(1):48-55. doi:10.1054/ijom.2003.0452

4. Toscano N, Holtzclaw D, Mazor Z, Rosen P, Horowitz R, Toffler M. Horizontal ridge augmentation utilizing a composite graft of demineralized freeze-dried allograft, mineralized cortical cancellous chips, and a biologically degradable thermoplastic carrier combined with a resorbable membrane: a retrospective evaluation of 73 consecutively treated cases from private practices. J Oral Implantol. 2010;36 (6):467-474. doi:10.1563/AAID-JOI-D-09-00100

5. Tolstunov L. Management of biomechanical complication of implant-supported restoration of a patient with combination syndrome: a case report. J Oral Maxillofac Surg. 2009;67(1):178-188. doi:10.1016/j.joms.2008.09.013

6. Gomes KU, Carlini JL, Biron C, Rapoport A, Dedivitis RA. Use of allogeneic bone graft in maxillary reconstruction for installation of dental implants. J Oral Maxillofac Surg. 2008;66(11):2335-2338. doi:10.1016/j.joms.2008.06.006

7. Wessing B, Lettner S, Zechner W. Guided bone regeneration with collagen membranes and particulate graft materials: a systematic review and meta-analysis. Int J Oral Maxillofac Implants. 2018;33 (1):87-100. doi:10.11607/jomi.5461

8. Rocchietta I, Simion M, Hoffmann M, Trisciuoglio D, Benigni M, Dahlin C. Vertical bone augmentation with an autogenous block or particles in combination with guided bone regeneration: a clinical and histological preliminary study in humans. Clin Implant Dent Relat Res. 2016;18(1):19-29. doi:10.1111/cid.12267
Clinical, Cosmetic and Investigational Dentistry

\section{Publish your work in this journal}

Clinical, Cosmetic and Investigational Dentistry is an international, peer-reviewed, open access, online journal focusing on the latest clinical and experimental research in dentistry with specific emphasis on cosmetic interventions. Innovative developments in dental materials, techniques and devices that improve outcomes and patient satisfaction and preference will be highlighted. The manuscript management system is completely online and includes a very quick and fair peer-review system, which is all easy to use. Visit http://www.dovepress.com/testimonials.php to read real quotes from published authors. 